\title{
Stabilization of Hybrid Systems by Feedback Control based on Discrete-time State Observations
}

\author{
Surong You ${ }^{1}$, Wei Liu ${ }^{2}$, Jianqiu Lu ${ }^{3}$, Xuerong Mao ${ }^{3 *}$ Qinwei Qiu ${ }^{4}$ \\ ${ }^{1}$ Department of Applied Mathematics, \\ Donghua Univerisity, Shanghai 201620, China. \\ ${ }^{2}$ Department of Mathematical Sciences, \\ Loughborough University, Loughborough, Leicestershire, LE11 3TU, U.K. \\ ${ }^{3}$ Department of Mathematics and Statistics, \\ University of Strathclyde, Glasgow G1 1XH, U.K. \\ ${ }^{4}$ College of Information Sciences and Technology, \\ Donghua Univerisity, Shanghai 201620, China.
}

\begin{abstract}
Recently, Mao [19] initiates the study the mean-square exponential stabilization of continuous-time hybrid stochastic differential equations by feedback controls based on discrete-time state observations. Mao [19] also obtains an upper bound on the duration $\tau$ between two consecutive state observations. However, it is due to the general technique used there that the bound on $\tau$ is not very sharp. In this paper, we will be able to establish a better bound on $\tau$ making use of Lyapunov functionals. We will not only discuss the stabilization in the sense of exponential stability (as Mao [19] does) but also in other sense of $H_{\infty}$ stability or asymptotic stability. We will not only consider the mean square stability but also the almost sure stability.
\end{abstract}

Key words: $H_{\infty}$ stability, asymptotic stability, exponential stability, feedback control, discrete-time state observation.

\section{Introduction}

An important class of hybrid systems is the calss of hybrid stochastic differential equations (SDEs) (also known as SDEs with Markovian switching). Indeed, hybrid SDEs have been used widely in many branches of science and industry to model systems where they may experience abrupt changes in their structure and parameters. One of the important issues in the study of hybrid SDEs is the automatic control, with consequent emphasis being placed on the asymptotic analysis of stability [3, 12, 24, 21, 22, 27, 28, 29, 30, 35]. In particular, [16,17] are two of most cited papers (Google citations 464 and 286,respectively) while [23] is the first book in this area (Google citation 559).

${ }^{*}$ Corresponding author. E-mail: x.mao@strath.ac.uk 
Recently, Mao [19] investigates the following stabilization problem by a feedback control based on the discrete-time state observations: Consider an unstable hybrid SDE

$$
d x(t)=f(x(t), r(t), t) d t+g(x(t), r(t), t) d w(t),
$$

where $x(t) \in R^{n}$ is the state, $w(t)=\left(w_{1}(t), \cdots, w_{m}(t)\right)^{T}$ is an $m$-dimensional Brownian motion, $r(t)$ is a Markov chain (please see Section 2 for the formal definitions) which represents the system mode, and the SDE is in the Itô sense. If this given hybrid SDE is not stable, it is traditional (or regular) to design a feedback control $u(x(t), r(t), t)$ in order for the controlled system

$$
\begin{aligned}
d x(t) & =(f(x(t), r(t), t)+u(x(t), r(t), t)) d t \\
& +g(x(t), r(t), t) d w(t)
\end{aligned}
$$

to become stable. Such a regular feedback control requires the continuous observations of the state $x(t)$ for all $t \geq 0$. This is of course expensive and sometimes not possible as the observations are often of discrete-time. It is therefore more reasonable and practical to design a feedback control $u(x([t / \tau] \tau), r(t), t)$ based on the discrete-time observations of the state $x(t)$ at times $0, \tau, 2 \tau, \cdots$ so that the controlled system

$$
\begin{aligned}
d x(t) & =(f(x(t), r(t), t)+u(x([t / \tau] \tau), r(t), t)) d t \\
& +g(x(t), r(t), t) d w(t)
\end{aligned}
$$

becomes stable, where $\tau>0$ is a constant and $[t / \tau]$ is the integer part of $t / \tau$. This is significantly different from the stabilization by a continuous-time (regular) feedback control $u(x(t), r(t), t)$. The regular feedback control requires the continuous observations of the state $x(t)$ for all $t \geq 0$, while the feedback control $u(x([t / \tau] \tau), r(t), t)$ needs only the discrete-time observations of the state $x(t)$ at times $0, \tau, 2 \tau, \cdots$. The latter is clearly more realistic and costs less in practice. To the best knowledge of the authors, Mao [19] is the first paper that studies this stabilization problem by feedback controls based on the discrete-time state observations in the area of SDEs, although the corresponding problem for the deterministic differential equations has been studied by many authors (see e.g. $[1,4,5,8,9])$.

Mao [19] shows that, under the global Lipschitz condition, if the continuous-time controlled SDE (1.2) is mean-square exponentially stable, then so is the discrete-timestate feedback controlled system (1.3) provided $\tau$ is sufficiently small. This is of course a very general result. However, it is due to the general technique used there that the bound on $\tau$ is not very sharp. In this paper, we will use the method of the Lyapunov functionals to study the stabilization problem. We will be able to improve the bound on $\tau$ significantly. The key features which differ from those in Mao [19] are as follows:

- Mao [19] has only discussed the stabilization in the sense of mean square exponential stability. In this paper, in addition to the mean square exponential stability, we will investigate the stabilization in the sense of $H_{\infty}$ stability as well as asymptotic stability. We will not only consider the mean square stability but also the almost sure stability, and the proof of the later is much more technical than that of former (please see the proof of Theorem 3.4 below).

- The key condition imposed in Mao [19] is the global Lipschitz condition on the coefficients of the underlying SDEs, while in this paper we only require a local Lipschitz condition and hence our new theory is applicable in much more general fashion. 
- The key technique in Mao [19] is to compare the discrete-time-state feedback controlled system (1.3) with the continuous-time controlled SDE (1.2) and then prove the stability of system (1.3) by making use of the stability of the SDE (1.2). However, in this paper, we will work directly on the discrete-time-state feedback controlled system (1.3) itself using the method of the Lyapunov functionals. To cope with the mixture of the continuous-time state $x(t)$ and the discrete-time state $x([t / \tau] \tau)$ in the same system, we have developed some new techniques.

Let us begin to develop these new techniques and to establish our new theory.

\section{Notation and Stabilization Problem}

Throughout this paper, unless otherwise specified, we let $\left(\Omega, \mathcal{F},\left\{\mathcal{F}_{t}\right\}_{t \geq 0}, \mathbb{P}\right)$ be a complete probability space with a filtration $\left\{\mathcal{F}_{t}\right\}_{t \geq 0}$ satisfying the usual conditions (i.e. it is increasing and right continuous while $\mathcal{F}_{0}$ contains all $\mathbb{P}$-null sets $)$. Let $w(t)=\left(w_{1}(t), \cdots, w_{m}(t)\right)^{T}$ be an $m$-dimensional Brownian motion defined on the probability space. If $A$ is a vector or matrix, its transpose is denoted by $A^{T}$. If $x \in R^{n}$, then $|x|$ is its Euclidean norm. If $A$ is a matrix, we let $|A|=\sqrt{\operatorname{trace}\left(A^{T} A\right)}$ be its trace norm and $\|A\|=\max \{|A x|:|x|=1\}$ be the operator norm. If $A$ is a symmetric matrix $\left(A=A^{T}\right)$, denote by $\lambda_{\min }(A)$ and $\lambda_{\max }(A)$ its smallest and largest eigenvalue, respectively. By $A \leq 0$ and $A<0$, we mean $A$ is non-positive and negative definite, respectively. If both $a, b$ are real numbers, then $a \vee b=\min \{a, b\}$ and $a \wedge b=\max \{a, b\}$. If $A$ is a subset of $\Omega$, denote by $I_{A}$ its indicator function; that is $I_{A}(\omega)=1$ when $\omega \in A$ and 0 otherwise.

Let $r(t), t \geq 0$, be a right-continuous Markov chain on the probability space taking values in a finite state space $S=\{1,2, \cdots, N\}$ with generator $\Gamma=\left(\gamma_{i j}\right)_{N \times N}$ given by

$$
\mathbb{P}\{r(t+\Delta)=j \mid r(t)=i\}= \begin{cases}\gamma_{i j} \Delta+o(\Delta) & \text { if } i \neq j \\ 1+\gamma_{i i} \Delta+o(\Delta) & \text { if } i=j\end{cases}
$$

where $\Delta>0$. Here $\gamma_{i j} \geq 0$ is the transition rate from $i$ to $j$ if $i \neq j$ while

$$
\gamma_{i i}=-\sum_{j \neq i} \gamma_{i j}
$$

We assume that the Markov chain $r(\cdot)$ is independent of the Brownian motion $w(\cdot)$.

Consider an $n$-dimensional controlled hybrid SDE

$$
d x(t)=\left(f(x(t), r(t), t)+u\left(x\left(\delta_{t}\right), r(t), t\right)\right) d t+g(x(t), r(t), t) d w(t)
$$

on $t \geq 0$, with initial data $x(0)=x_{0} \in R^{n}$ and $r(0)=r_{0} \in S$ at time zero. Here

$$
f, u: R^{n} \times S \times R_{+} \rightarrow R^{n} \quad \text { and } \quad g: R^{n} \times S \times R_{+} \rightarrow R^{n \times m},
$$

while $\tau>0$ and

$$
\delta_{t}=[t / \tau] \tau,
$$

in which $[t / \tau]$ is the integer part of $t / \tau$. Our aim here is to design the feedback control $u\left(x\left(\delta_{t}\right), r(t), t\right)$ so that this controlled hybrid SDE becomes mean-square asymptotically stable, though the given uncontrolled system

$$
d x(t)=f(x(t), r(t), t) d t+g(x(t), r(t), t) d w(t)
$$


may not be stable. We observe that the feedback control $u\left(x\left(\delta_{t}\right), r(t), t\right)$ is designed based on the discrete-time state observations $x(0), x(\tau), x(2 \tau), \cdots$, though the given hybrid SDE (2.3) is of continuous-time. In this paper we impose the following standing hypotheses.

Assumption 2.1 Assume that the coefficients $f$ and $g$ are all locally Lipschitz continuous (see e.g. [13, 14, 15, 23]). Moreover, they satisfy the following linear growth condition

$$
|f(x, i, t)| \leq K_{1}|x| \quad \text { and } \quad|g(x, i, t)| \leq K_{2}|x|
$$

for all $(x, i, t) \in R^{n} \times S \times R_{+}$, where both $K_{1}$ and $K_{2}$ are positive numbers.

We observe that (2.4) forces

$$
f(0, i, t)=0, \quad g(0, i, t)=0
$$

for all $(i, t) \in S \times R_{+}$. This is of course for the stability purpose of this paper. For a technical reason, we require a global Lipschitz condition on the controller function $u$. More precisely, we impose the following hypothesis.

Assumption 2.2 Assume that there exists a positive constant $K_{3}$ such that

$$
|u(x, i, t)-u(y, i, t)| \leq K_{3}|x-y|
$$

for all $(x, y, i, t) \in R^{n} \times R^{n} \times S \times R_{+}$. Moreover,

$$
u(0, i, t)=0
$$

for all $(i, t) \in S \times R_{+}$.

Once again, condition (2.7) is for the stability purpose of this paper. We also see that Assumption 2.2 implies the following linear growth condition on the controller function

$$
|u(x, i, t)| \leq K_{3}|x|
$$

for all $(x, i, t) \in R^{n} \times S \times R_{+}$.

We observe that equation (2.1) is in fact a stochastic differential delay equation (SDDE) with a bounded variable delay. Indeed, if we define the bounded variable delay $\zeta:[0, \infty) \rightarrow[0, \tau]$ by

$$
\zeta(t)=t-k \tau \quad \text { for } k \tau \leq t<(k+1) \tau, k=0,1,2, \cdots,
$$

then equation (2.1) can be written as

$$
d x(t)=(f(x(t), r(t), t)+u(x(t-\zeta(t)), r(t), t)) d t+g(x(t), r(t), t) d w(t) .
$$

It is therefore known (see e.g. [23]) that under Assumptions 2.1 and 2.2, the SDDE (2.9) (namely the controlled system (2.1)) has a unique solution $x(t)$ such that $\mathbb{E}|x(t)|^{2}<\infty$ for all $t \geq 0$. Of course, we should point out that equation (2.9) is a special SDDE in the sense we need to know only the initial data $x(0)$ and $r(0)$ at $t=0$ in order to determine the unique solution $x(t)$ on $t \geq 0$. However, if we are given data $x(s)$ and $r(s)$ for some $s \in(k \tau,(k+1) \tau)$, we will not be able to determine the solution $x(t)$ on $t \geq s$ unless we also know $x(k \tau)$. 
The observation above also shows that the stability and stabilization problem of equation (2.1) can be regarded as the problem of the hybrid SDDE (2.9) with a bounded variable delay. On the other hand, as far as the authors know, the existing results on the stability of the hybrid SDDE require the bounded variable delay be differentiable and the derivative be less than one (see e.g. [11, p.182] or [23, p.285]). However, the bounded variable delay $\zeta(t)$ defined above is not differentiable when $t=k \tau, k=1,2, \cdots$, while its derivative $d \zeta(t) / d t=1$ for $t \in((k-1) \tau, k \tau)$. Therefore, the existing results on the stability of the hybrid SDDEs are not applicable here and we need to develop our new theory.

\section{$3 \quad$ Asymptotic Stabilization}

For our stabilization purpose related to the controlled system (2.1) we will use a Lyapunov functional on the segments $\hat{x}_{t}:=\{x(t+s):-2 \tau \leq s \leq 0\}$ and $\hat{r}_{t}:=\{r(t+s):-2 \tau \leq$ $s \leq 0\}$ for $t \geq 0$. For $\hat{x}_{t}$ and $\hat{r}_{t}$ to be well defined for $0 \leq t<2 \tau$, we set $x(s)=x_{0}$ and $r(s)=r_{0}$ for $-2 \tau \leq s \leq 0$. The Lyapunov functional used in this paper will be of the form

$$
\begin{aligned}
& V\left(\hat{x}_{t}, \hat{r}_{t}, t\right)=U(x(t), r(t), t) \\
& +\theta \int_{t-\tau}^{t} \int_{s}^{t}\left[\tau\left|f(x(v), r(v), v)+u\left(x\left(\delta_{v}\right), r(v), v\right)\right|^{2}+|g(x(v), r(v), v)|^{2}\right] d v d s
\end{aligned}
$$

for $t \geq 0$, where $\theta$ is a positive number to be determined later and we set

$$
f(x, i, s)=f(x, i, 0), \quad u(x, i, s)=u(x, i, 0), \quad g(x, i, s)=f(x, i, 0)
$$

for $(x, i, s) \in R^{n} \times S \times[-2 \tau, 0)$. Of course, the functional above uses $r(u)$ only on $t-\tau \leq u \leq t$ so we could have defined $\hat{r}_{t}:=\{r(t+s):-\tau \leq s \leq 0\}$. But, to be consistent with the definition of $\hat{x}_{t}$, we define $\hat{r}_{t}$ as above and this does not lose any generality. We also require $U \in C^{2,1}\left(R^{n} \times S \times R_{+} ; R_{+}\right)$, the family of non-negative functions $U(x, i, t)$ defined on $(x, i, t) \in R^{n} \times S \times R_{+}$which are continuously twice differentiable in $x$ and once in $t$. For $U \in C^{2,1}\left(R^{n} \times S \times R_{+} ; R_{+}\right)$, let us define $\mathcal{L} U: R^{n} \times S \times R_{+} \rightarrow R$ by

$$
\begin{aligned}
\mathcal{L} U(x, i, t) & =U_{t}(x, i, t)+U_{x}(x, i, t)[f(x, i, t)+u(x, i, t)] \\
& +\frac{1}{2} \operatorname{trace}\left[g^{T}(x, i, t) U_{x x}(x, i, t) g(x, i, t)\right]+\sum_{j=1}^{N} \gamma_{i j} U(x, j, t),
\end{aligned}
$$

where

$$
U_{t}(x, i, t)=\frac{\partial U(x, i, t)}{\partial t}, \quad U_{x}(x, i, t)=\left(\frac{\partial U(x, i, t)}{\partial x_{1}}, \cdots, \frac{\partial U(x, i, t)}{\partial x_{n}}\right)
$$

and

$$
U_{x x}(x, i, t)=\left(\frac{\partial^{2} U(x, i, t)}{\partial x_{i} \partial x_{j}}\right)_{n \times n} .
$$

Let us now impose a new assumption on $U$.

Assumption 3.1 Assume that there is a function $U \in C^{2,1}\left(R^{n} \times S \times R_{+} ; R_{+}\right)$and two positive numbers $\lambda_{1}, \lambda_{2}$ such that

$$
\mathcal{L} U(x, i, t)+\lambda_{1}\left|U_{x}(x, i, t)\right|^{2} \leq-\lambda_{2}|x|^{2}
$$

for all $(x, i, t) \in R^{n} \times S \times R_{+}$. 
Let us comment on this assumption. Condition (3.3) implies

$$
\mathcal{L} U(x, i, t) \leq-\lambda_{2}|x|^{2}
$$

which guarantees the asymptotic stability (in mean square etc.) of the controlled system (1.2). In other words, the continuous-time feedback control $u(x(t), r(t), t)$ will stabilize the system. However, in order for the discrete-time feedback control $u\left(x\left(\delta_{t}\right), r(t), t\right)$ to do the job, we need a slightly stronger condition, namely we add a new term $\lambda_{1}\left|U_{x}(x, i, t)\right|^{2}$ into the left-hand-side of (3.4) to form (3.3). As demonstrated in Sections 5 and 6 later, we will see this is quite easy to achieve by choosing $\lambda_{1}$ sufficiently small when the derivative vector $U_{x}(x, i, t)$ is bounded by a linear function of $x$. We can now state our first result.

Theorem 3.2 Let Assumptions 2.1, 2.2 and 3.1 hold. If $\tau>0$ is sufficiently small for

$$
\lambda_{2}>\frac{\tau K_{3}^{2}}{\lambda_{1}}\left[2 \tau\left(K_{1}^{2}+2 K_{3}^{2}\right)+K_{2}^{2}\right] \quad \text { and } \quad \tau \leq \frac{1}{4 K_{3}},
$$

then the controlled system (2.1) is $H_{\infty}$-stable in the sense that

$$
\int_{0}^{\infty} \mathbb{E}|x(s)|^{2} d s<\infty
$$

for all initial data $x_{0} \in R^{n}$ and $r_{0} \in S$.

Proof. Fix any $x_{0} \in R^{n}$ and $r_{0} \in S$. Applying the generalized Itô formula (see e.g. $[17,23])$ to the Lyapunov functional defined by (3.1) yields

$$
d V\left(\hat{x}_{t}, \hat{r}_{t}, t\right)=L V\left(\hat{x}_{t}, \hat{r}_{t}, t\right) d t+d M(t)
$$

for $t \geq 0$, where $M(t)$ is a continuous martingale with $M(0)=0$ (the explicit form of $M(t)$ is of no use in this paper so we do not state it here) and

$$
\begin{aligned}
& L V\left(\hat{x}_{t}, \hat{r}_{t}, t\right) \\
& =U_{t}(x(t), r(t), t)+U_{x}(x(t), r(t), t)\left[f(x(t), r(t), t)+u\left(x\left(\delta_{t}\right), r(t), t\right)\right] \\
& +\frac{1}{2} \operatorname{trace}\left[g^{T}(x(t), r(t), t) U_{x x}(x(t), r(t), t) g(x(t), r(t), t)\right] \\
& +\sum_{j=1}^{N} \gamma_{r(t), j} U(x(t), j, t) \\
& +\theta \tau\left[\tau\left|f(x(t), r(t), t)+u\left(x\left(\delta_{t}\right), r(t), t\right)\right|^{2}+|g(x(t), r(t), t)|^{2}\right] \\
& -\theta \int_{t-\tau}^{t}\left[\tau\left|f(x(s), r(s), s)+u\left(x\left(\delta_{s}\right), r(s), s\right)\right|^{2}+|g(x(s), r(s), s)|^{2}\right] d s .
\end{aligned}
$$

To see why (3.7) holds, we regard the solution $x(t)$ of equation (2.1) as an Itô process and apply the generalized Itô formula (see e.g. $[17,23])$ to $U(x(t), r(t), t)$ to get

$$
\begin{aligned}
d U(x(t), r(t), t) & =\left(U_{t}(x(t), r(t), t)+U_{x}(x(t), r(t), t)\left[f(x(t), r(t), t)+u\left(x\left(\delta_{t}\right), r(t), t\right)\right]\right. \\
& +\frac{1}{2} \operatorname{trace}\left[g^{T}(x(t), r(t), t) U_{x x}(x(t), r(t), t) g(x(t), r(t), t)\right] \\
& \left.+\sum_{j=1}^{N} \gamma_{r(t), j} U(x(t), j, t)\right) d t+d M(t) .
\end{aligned}
$$


On the other hand, the fundamental theory of calculus shows

$$
\begin{aligned}
& d\left(\int_{t-\tau}^{t} \int_{s}^{t}\left[\tau\left|f(x(v), r(v), v)+u\left(x\left(\delta_{v}\right), r(v), v\right)\right|^{2}+|g(x(v), r(v), v)|^{2}\right] d v d s\right) \\
& =\left(\tau\left[\tau\left|f(x(t), r(t), t)+u\left(x\left(\delta_{t}\right), r(t), t\right)\right|^{2}+|g(x(t), r(t), t)|^{2}\right]\right. \\
& \left.-\int_{t-\tau}^{t}\left[\tau\left|f(x(s), r(s), s)+u\left(x\left(\delta_{s}\right), r(s), s\right)\right|^{2}+|g(x(s), r(s), s)|^{2}\right] d s\right) d t
\end{aligned}
$$

Combining these two equalities gives (3.7).

Recalling (3.2), we can re-write (3.8) as

$$
\begin{aligned}
& L V\left(\hat{x}_{t}, \hat{r}_{t}, t\right) \\
& =\mathcal{L} U(x(t), r(t), t)-U_{x}(x(t), r(t), t)\left[u(x(t), r(t), t)-u\left(x\left(\delta_{t}\right), r(t), t\right)\right] \\
& +\theta \tau\left[\tau\left|f(x(t), r(t), t)+u\left(x\left(\delta_{t}\right), r(t), t\right)\right|^{2}+|g(x(t), r(t), t)|^{2}\right] \\
& -\theta \int_{t-\tau}^{t}\left[\tau\left|f(x(s), r(s), s)+u\left(x\left(\delta_{s}\right), r(s), s\right)\right|^{2}+|g(x(s), r(s), s)|^{2}\right] d s .
\end{aligned}
$$

But, by Assumption 2.2,

$$
\begin{aligned}
& -U_{x}(x(t), r(t), t)\left[u(x(t), r(t), t)-u\left(x\left(\delta_{t}\right), r(t), t\right)\right] \\
\leq & \lambda_{1}\left|U_{x}(x(t), r(t), t)\right|^{2}+\frac{1}{4 \lambda_{1}}\left|u(x(t), r(t), t)-u\left(x\left(\delta_{t}\right), r(t), t\right)\right|^{2} \\
\leq & \lambda_{1}\left|U_{x}(x(t), r(t), t)\right|^{2}+\frac{K_{3}^{2}}{4 \lambda_{1}}\left|x(t)-x\left(\delta_{t}\right)\right|^{2} .
\end{aligned}
$$

Moreover, by Assumptions 2.1 and 2.2, we have

$$
\begin{aligned}
& \theta \tau\left[\tau\left|f(x(t), r(t), t)+u\left(x\left(\delta_{t}\right), r(t), t\right)\right|^{2}+|g(x(t), r(t), t)|^{2}\right] \\
\leq & \theta \tau\left[2 \tau\left(K_{1}^{2}|x(t)|^{2}+K_{3}^{2}\left|x\left(\delta_{t}\right)\right|^{2}\right)+K_{2}^{2}|x(t)|^{2}\right] \\
\leq & \theta \tau\left[2 \tau\left(K_{1}^{2}+2 K_{3}^{2}\right)+K_{2}^{2}\right]|x(t)|^{2}+4 \theta \tau^{2} K_{3}^{2}\left|x(t)-x\left(\delta_{t}\right)\right|^{2} .
\end{aligned}
$$

Substituting (3.10) and (3.11) yields

$$
\begin{aligned}
& L V\left(\hat{x}_{t}, \hat{r}_{t}, t\right) \leq \mathcal{L} U(x(t), r(t), t)+\lambda_{1}\left|U_{x}(x(t), r(t), t)\right|^{2} \\
& +\theta \tau\left[2 \tau\left(K_{1}^{2}+2 K_{3}^{2}\right)+K_{2}^{2}\right]|x(t)|^{2}+\left(\frac{K_{3}^{2}}{4 \lambda_{1}}+4 \theta \tau^{2} K_{3}^{2}\right)\left|x(t)-x\left(\delta_{t}\right)\right|^{2} \\
& \quad-\theta \int_{t-\tau}^{t}\left[\tau\left|f(x(s), r(s), s)+u\left(x\left(\delta_{s}\right), r(s), s\right)\right|^{2}+|g(x(s), r(s), s)|^{2}\right] d s .
\end{aligned}
$$

It then follows from (3.12) and Assumption 3.1 that

$$
\begin{aligned}
& L V\left(\hat{x}_{t}, \hat{r}_{t}, t\right) \leq-\lambda|x(t)|^{2}+\left(\frac{K_{3}^{2}}{4 \lambda_{1}}+4 \theta \tau^{2} K_{3}^{2}\right)\left|x(t)-x\left(\delta_{t}\right)\right|^{2} \\
& \quad-\theta \int_{t-\tau}^{t}\left[\tau\left|f(x(s), r(s), s)+u\left(x\left(\delta_{s}\right), r(s), s\right)\right|^{2}+|g(x(s), r(s), s)|^{2}\right] d s,
\end{aligned}
$$

where

$$
\lambda=\lambda(\theta, \tau):=\lambda_{2}-\theta \tau\left[2 \tau\left(K_{1}^{2}+2 K_{3}^{2}\right)+K_{2}^{2}\right] .
$$


Noting that $t-\delta_{t} \leq \tau$ for all $t \geq 0$, we can show easily from (2.1) that

$$
\begin{aligned}
& \mathbb{E}\left|x(t)-x\left(\delta_{t}\right)\right|^{2} \\
\leq & 2 \mathbb{E} \int_{\delta_{t}}^{t}\left[\tau\left|f(x(s), r(s), s)+u\left(x\left(\delta_{s}\right), r(s), s\right)\right|^{2}+|g(x(s), r(s), s)|^{2}\right] d s .
\end{aligned}
$$

Let us now choose

$$
\theta=\frac{K_{3}^{2}}{\lambda_{1}} \quad \text { and } \quad \tau \leq \frac{1}{4 K_{3}} .
$$

It then follows from (3.13) and (3.15) that

$$
\mathbb{E}\left(L V\left(\hat{x}_{t}, \hat{r}_{t}, t\right)\right) \leq-\lambda \mathbb{E}|x(t)|^{2},
$$

and by condition (3.5) we have $\lambda>0$. By (3.7), we hence have

$$
\mathbb{E}\left(V\left(\hat{x}_{t}, \hat{r}_{t}, t\right)\right) \leq C_{1}-\lambda \int_{0}^{t} \mathbb{E}|x(s)|^{2} d s
$$

for $t \geq 0$, where

$$
\begin{aligned}
C_{1} & =V\left(\hat{x}_{0}, \hat{r}_{0}, 0\right) \\
& =U\left(x_{0}, r_{0}, 0\right)+0.5 \theta \tau^{2}\left[\tau\left|f\left(x_{0}, r_{0}, 0\right)+u\left(x_{0}, r_{0}, 0\right)\right|^{2}+\left|g\left(x_{0}, r_{0}, 0\right)\right|^{2}\right],
\end{aligned}
$$

so $C_{1}$ is a positive number. It follows from (3.18) immediately that

$$
\int_{0}^{\infty} \mathbb{E}|x(s)|^{2} d s \leq C_{1} / \lambda
$$

This implies the desired assertion (3.6).

In general, it does not follow from (3.6) that $\lim _{t \rightarrow \infty} \mathbb{E}\left(|x(t)|^{2}\right)=0$. But, in our case, this is possible. We state this as our second result.

Theorem 3.3 Under the same assumptions of Theorem 3.2, the solution of the controlled system (2.1) satisfies

$$
\lim _{t \rightarrow \infty} \mathbb{E}|x(t)|^{2}=0
$$

for all initial data $x_{0} \in R^{n}$ and $r_{0} \in S$. That is, the controlled system (2.1) is asymptotically stable in mean square.

Proof. Again, fix any $x_{0} \in R^{n}$ and $r_{0} \in S$. By the Itô formula, we have

$$
\mathbb{E}\left(|x(t)|^{2}\right)=\left|x_{0}\right|^{2}+\mathbb{E} \int_{0}^{t}\left(2 x(s)\left[f(x(s), r(s), s)+u\left(x\left(\delta_{s}\right), r(s), s\right)\right]+|g(x(s), r(s), s)|^{2}\right) d t
$$

for all $t \geq 0$. By Assumptions 2.1 and 2.2, it is easy to show that

$$
\mathbb{E}|x(t)|^{2} \leq\left|x_{0}\right|^{2}+C \int_{0}^{t} \mathbb{E}|x(s)|^{2} d s+C \int_{0}^{t} \mathbb{E}\left|x(s)-x\left(\delta_{s}\right)\right|^{2} d s,
$$

where, and in the remaining part of this paper, $C$ denotes a positive constant that may change from line to line but its special form is of no use. For any $s \geq 0$, there is a unique 
integer $v \geq 0$ for $s \in[v \tau,(v+1) \tau)$. Moreover, $\delta_{z}=v \tau$ for $z \in[v \tau, s]$. It follows from (2.1) that

$$
\begin{aligned}
& x(s)-x\left(\delta_{s}\right)=x(s)-x(v \tau) \\
= & \int_{v \tau}^{s}[f(x(z), r(z), z)+u(x(v \tau), r(z), z)] d z+\int_{v \tau}^{s} g(x(z), r(z), z) d w(z) .
\end{aligned}
$$

By Assumptions 2.1 and 2.2, we can then derive

$$
\begin{aligned}
& \mathbb{E}\left|x(s)-x\left(\delta_{s}\right)\right|^{2} \\
\leq & 3\left(\tau K_{1}^{2}+K_{2}^{2}\right) \int_{v \tau}^{s} \mathbb{E}|x(z)|^{2} d z+3 \tau^{2} K_{3}^{2} \mathbb{E}|x(v \tau)|^{2} \\
\leq & 3\left(\tau K_{1}^{2}+K_{2}^{2}\right) \int_{\delta_{s}}^{s} \mathbb{E}|x(z)|^{2} d z+6 \tau^{2} K_{3}^{2}\left(\mathbb{E}|x(s)|^{2}+\mathbb{E}\left|x(s)-x\left(\delta_{s}\right)\right|^{2}\right) .
\end{aligned}
$$

Noting that $6 \tau^{2} K_{3}^{2}<1$ by condition (3.5), we hence have

$$
\mathbb{E}\left|x(s)-x\left(\delta_{s}\right)\right|^{2} \leq \frac{3\left(\tau K_{1}^{2}+K_{2}^{2}\right)}{1-6 \tau^{2} K_{3}^{2}} \int_{\delta_{s}}^{s} \mathbb{E}|x(z)|^{2} d z+\frac{6 \tau^{2} K_{3}^{2}}{1-6 \tau^{2} K_{3}^{2}} \mathbb{E}|x(s)|^{2} .
$$

Substituting this into (3.20) yields

$$
\mathbb{E}|x(t)|^{2} \leq\left|x_{0}\right|^{2}+C \int_{0}^{t} \mathbb{E}|x(s)|^{2} d s+C \int_{0}^{t} \int_{\delta_{s}}^{s} \mathbb{E}|x(z)|^{2} d z d s .
$$

But, it is easy to derive that

$$
\begin{aligned}
& \int_{0}^{t} \int_{\delta_{s}}^{s} \mathbb{E}|x(z)|^{2} d z d s \leq \int_{0}^{t} \int_{s-\tau}^{s} \mathbb{E}|x(z)|^{2} d z d s \\
& \leq \int_{-\tau}^{t} \mathbb{E}|x(z)|^{2} \int_{z}^{z+\tau} d s d z \leq \tau \int_{-\tau}^{t} \mathbb{E}|x(z)|^{2} d z
\end{aligned}
$$

Substituting this into (3.22) and then applying Theorem 3.2, we obtain that

$$
\mathbb{E}|x(t)|^{2} \leq C \quad \forall t \geq 0
$$

By the Itô formula, we have

$$
\begin{aligned}
& \mathbb{E}\left|x\left(t_{2}\right)\right|^{2}-\mathbb{E}\left|x\left(t_{1}\right)\right|^{2} \\
= & \mathbb{E} \int_{t_{1}}^{t_{2}}\left(2 x(t)\left[f(x(t), r(t), t)+u\left(x\left(\delta_{t}\right), r(t), t\right)\right]+|g(x(t), r(t), t)|^{2}\right) d t
\end{aligned}
$$

for any $0 \leq t_{1}<t_{2}<\infty$. Using (3.23) and Assumptions 2.1 and 2.2, we can then easily show that

$$
\left.|\mathbb{E}| x\left(t_{2}\right)\right|^{2}-\mathbb{E}\left|x\left(t_{1}\right)\right|^{2} \mid \leq C\left(t_{2}-t_{1}\right) .
$$

That is, $\mathbb{E}|x(t)|^{2}$ is uniformly continuous in $t$ on $R_{+}$. It then follows from (3.6) that $\lim _{t \rightarrow \infty} \mathbb{E}|x(t)|^{2}=0$ as required.

In general, we cannot imply $\lim _{t \rightarrow \infty}|x(t)|=0$ a.s. from $\lim _{t \rightarrow \infty} \mathbb{E}\left(|x(t)|^{2}\right)=0$. But, in our case, this is once again possible. We state this as our third result. 
Theorem 3.4 Under the same assumptions of Theorem 3.2, the solution of the controlled system (2.1) satisfies

$$
\lim _{t \rightarrow \infty} x(t)=0 \quad \text { a.s. }
$$

for all initial data $x_{0} \in R^{n}$ and $r_{0} \in S$. That is, the controlled system (2.1) is almost surely asymptotically stable.

Proof. The proof is very technical so we divide it into three steps.

Step 1. Again we fix any $x_{0} \in R^{n}$ and $r_{0} \in S$. It follows from Theorem 3.3 and the well known Fubini theorem that

$$
\mathbb{E} \int_{0}^{\infty}|x(t)|^{2} d t<\infty
$$

This implies

$$
\int_{0}^{\infty}|x(t)|^{2} d t<\infty \quad \text { a.s. }
$$

We must therefore have

$$
\liminf _{t \rightarrow \infty}|x(t)|=0 \quad \text { a.s. }
$$

We now claim that

$$
\lim _{t \rightarrow \infty}|x(t)|=0 \quad \text { a.s. }
$$

If this is false, then

$$
\mathbb{P}\left(\limsup _{t \rightarrow \infty}|x(t)|>0\right)>0
$$

We hence can find a positive number $\varepsilon$, sufficiently small, for

$$
\mathbb{P}\left(\Omega_{1}\right) \geq 3 \varepsilon
$$

where

$$
\Omega_{1}=\left\{\limsup _{t \rightarrow \infty}|x(t)|>2 \varepsilon\right\}
$$

Step 2. Let $h>\left|x_{0}\right|$ be a number. Define the stopping time

$$
\beta_{h}=\inf \{t \geq 0:|x(t)| \geq h\}
$$

where throughout this paper we set $\inf \emptyset=\infty$ (in which $\emptyset$ denotes the empty set as usual). Then, by the Itô formula, we have

$$
\begin{aligned}
& \mathbb{E}\left|x\left(t \wedge \beta_{h}\right)\right|^{2} \\
& =\left|x_{0}\right|^{2}+\mathbb{E} \int_{0}^{t \wedge \beta_{h}}\left(2 x(s)\left[f(x(s), r(s), s)+u\left(x\left(\delta_{s}\right), r(s), s\right)\right]+|g(x(s), r(s), s)|^{2}\right) d t
\end{aligned}
$$

for all $t \geq 0$. By Assumptions 2.1 and 2.2 as well as Theorem 3.2, it is easy to show that

$$
\mathbb{E}\left|x\left(t \wedge \beta_{h}\right)\right|^{2} \leq C
$$

Hence

$$
h^{2} \mathbb{P}\left(\beta_{h} \leq t\right) \leq C
$$


Letting $t \rightarrow \infty$ and then choosing $h$ sufficiently large, we get

$$
\mathbb{P}\left(\beta_{h}<\infty\right) \leq \frac{C}{h^{2}} \leq \varepsilon
$$

This implies

$$
\mathbb{P}\left(\Omega_{2}\right) \geq 1-\varepsilon
$$

where

$$
\Omega_{2}=\{|x(t)|<h \text { for all } 0 \leq t<\infty\} .
$$

It then follows easily from (3.27) and (3.28) that

$$
\mathbb{P}\left(\Omega_{1} \cap \Omega_{2}\right) \geq 2 \varepsilon \text {. }
$$

Step 3. Define a sequence of stopping times:

$$
\begin{aligned}
\alpha_{1} & =\inf \left\{t \geq 0:|x(t)|^{2} \geq 2 \varepsilon\right\}, \\
\alpha_{2 i} & =\inf \left\{t \geq \alpha_{2 i-1}:|x(t)|^{2} \leq \varepsilon\right\}, \quad i=1,2, \cdots, \\
\alpha_{2 i+1} & =\inf \left\{t \geq \alpha_{2 i}:|x(t)|^{2} \geq 2 \varepsilon\right\}, \quad i=1,2, \cdots .
\end{aligned}
$$

We observe from (3.25) and the definitions of $\Omega_{1}$ and $\Omega_{2}$ that $\alpha_{2 i}<\infty$ whenever $\alpha_{2 i-1}<$ $\infty$, and moreover,

$$
\beta_{h}(\omega)=\infty \text { and } \alpha_{i}(\omega)<\infty \text { for all } i \geq 1 \text { whenever } \omega \in \Omega_{1} \cap \Omega_{2} .
$$

By (3.24), we derive

$$
\begin{aligned}
\infty & >\mathbb{E} \int_{0}^{\infty}|x(t)|^{2} d t \geq \sum_{i=1}^{\infty} \mathbb{E}\left(I_{\left\{\alpha_{2 i-1}<\infty, \beta_{h}=\infty\right\}} \int_{\alpha_{2 i-1}}^{\alpha_{2 i}}|x(t)|^{2} d t\right) \\
& \geq \varepsilon \sum_{i=1}^{\infty} \mathbb{E}\left(I_{\left\{\alpha_{2 i-1}<\infty, \beta_{h}=\infty\right\}}\left[\alpha_{2 i}-\alpha_{2 i-1}\right]\right) .
\end{aligned}
$$

Let use now define

$$
F(t)=f(x(t), r(t), t)+u\left(x\left(\delta_{t}\right), r(t), t\right) \quad \text { and } \quad G(t)=g(x(t), r(t), t)
$$

for $t \geq 0$. By Assumptions 2.1 and 2.2, we see that

$$
|F(t)|^{2} \wedge|G(t)|^{2} \leq K_{h} \quad \forall t \geq 0
$$

whenever $|x(t) \wedge| x\left(\delta_{t}\right) \mid \leq h$ (in particular, for $\omega \in \Omega_{2}$ ), where $K_{h}$ is a positive constant. By the Hölder inequality and the Doob martingale inequality, we then derive that, for any $T>0$,

$$
\begin{aligned}
& \mathbb{E}\left(I_{\left\{\beta_{h} \wedge \alpha_{2 i-1}<\infty\right\}} \sup _{0 \leq t \leq T}\left|x\left(\beta_{h} \wedge\left(\alpha_{2 i-1}+t\right)\right)-x\left(\beta_{h} \wedge \alpha_{2 i-1}\right)\right|^{2}\right) \\
\leq & 2 \mathbb{E}\left(I_{\left\{\beta_{h} \wedge \alpha_{2 i-1}<\infty\right\}} \sup _{0 \leq t \leq T}\left|\int_{\beta_{h} \wedge \alpha_{2 i-1}}^{\beta_{h} \wedge\left(\alpha_{2 i-1}+t\right)} F(s) d s\right|^{2}\right) \\
+ & 2 \mathbb{E}\left(I_{\left\{\beta_{h} \wedge \alpha_{2 i-1}<\infty\right\}} \sup _{0 \leq t \leq T}\left|\int_{\beta_{h} \wedge \alpha_{2 i-1}}^{\beta_{h} \wedge\left(\alpha_{2 i-1}+t\right)} G(s) d w(s)\right|^{2}\right) \\
\leq & 2 T \mathbb{E}\left(I_{\left\{\beta_{h} \wedge \alpha_{2 i-1}<\infty\right\}} \int_{\beta_{h} \wedge \alpha_{2 i-1}}^{\beta_{h} \wedge\left(\alpha_{2 i-1}+T\right)}|F(s)|^{2} d s\right) \\
+ & 8 \mathbb{E}\left(I_{\left\{\beta_{h} \wedge \alpha_{2 i-1}<\infty\right\}} \int_{\beta_{h} \wedge \alpha_{2 i-1}}^{\beta_{h} \wedge\left(\alpha_{2 i-1}+T\right)}|G(s)|^{2} d s\right) \\
\leq & 2 K_{h} T(T+4) .
\end{aligned}
$$


Let $\theta=\varepsilon /(2 h)$. It is easy to see that

$$
\left.|| x\right|^{2}-|y|^{2} \mid<\varepsilon \text { whenever }|x-y|<\theta,|x| \vee|y| \leq h \text {. }
$$

Choose $T$ sufficiently small for

$$
\frac{2 K_{h} T(T+4)}{\theta^{2}}<\varepsilon
$$

It then follows from (3.32) that

$$
\begin{aligned}
& \mathbb{P}\left(\left\{\beta_{h} \wedge \alpha_{2 i-1}<\infty\right\} \cap\left\{\sup _{0 \leq t \leq T}\left|x\left(\beta_{h} \wedge\left(\alpha_{2 i-1}+t\right)\right)-x\left(\beta_{h} \wedge \alpha_{2 i-1}\right)\right| \geq \theta\right\}\right) \\
& \quad \leq \frac{2 K_{h} T(T+4)}{\theta^{2}}<\varepsilon .
\end{aligned}
$$

Therefore

$$
\begin{aligned}
& \mathbb{P}\left(\left\{\alpha_{2 i-1}<\infty, \beta_{h}=\infty\right\} \cap\left\{\sup _{0 \leq t \leq T}\left|x\left(\alpha_{2 i-1}+t\right)-x\left(\alpha_{2 i-1}\right)\right| \geq \theta\right\}\right) \\
= & \mathbb{P}\left(\left\{\beta_{h} \wedge \alpha_{2 i-1}<\infty, \beta_{h}=\infty\right\} \cap\left\{\sup _{0 \leq t \leq T}\left|x\left(\beta_{h} \wedge\left(\alpha_{2 i-1}+t\right)\right)-x\left(\beta_{h} \wedge \alpha_{2 i-1}\right)\right| \geq \theta\right\}\right) \\
\leq & \mathbb{P}\left(\left\{\beta_{h} \wedge \alpha_{2 i-1}<\infty\right\} \cap\left\{\sup _{0 \leq t \leq T}\left|x\left(\beta_{h} \wedge\left(\alpha_{2 i-1}+t\right)\right)-x\left(\beta_{h} \wedge \alpha_{2 i-1}\right)\right| \geq \theta\right\}\right) \\
\leq & \varepsilon .
\end{aligned}
$$

Using (3.29) and (3.30), we then have

$$
\begin{aligned}
& \mathbb{P}\left(\left\{\alpha_{2 i-1}<\infty, \beta_{h}=\infty\right\} \cap\left\{\sup _{0 \leq t \leq T}\left|x\left(\alpha_{2 i-1}+t\right)-x\left(\alpha_{2 i-1}\right)\right|<\theta\right\}\right) \\
= & \mathbb{P}\left(\left\{\alpha_{2 i-1}<\infty, \beta_{h}=\infty\right\}\right) \\
- & \mathbb{P}\left(\left\{\alpha_{2 i-1}<\infty, \beta_{h}=\infty\right\} \cap\left\{\sup _{0 \leq t \leq T}\left|x\left(\alpha_{2 i-1}+t\right)-x\left(\alpha_{2 i-1}\right)\right| \geq \theta\right\}\right) \\
\geq & 2 \varepsilon-\varepsilon=\varepsilon .
\end{aligned}
$$

By (3.33), we get

$$
\begin{aligned}
& \mathbb{P}\left(\left\{\alpha_{2 i-1}<\infty, \beta_{h}=\infty\right\} \cap\left\{\left.\sup _{0 \leq t \leq T}|| x\left(\alpha_{2 i-1}+t\right)\right|^{2}-\left|x\left(\alpha_{2 i-1}\right)\right|^{2} \mid<\varepsilon\right\}\right) \\
\geq & \mathbb{P}\left(\left\{\alpha_{2 i-1}<\infty, \beta_{h}=\infty\right\} \cap\left\{\sup _{0 \leq t \leq T}\left|x\left(\alpha_{2 i-1}+t\right)-x\left(\alpha_{2 i-1}\right)\right|<\theta\right\}\right) \\
\geq & \varepsilon .
\end{aligned}
$$

Set

$$
\hat{\Omega}_{i}=\left\{\left.\sup _{0 \leq t \leq T}|| x\left(\alpha_{2 i-1}+t\right)\right|^{2}-\left|x\left(\alpha_{2 i-1}\right)\right|^{2} \mid<\varepsilon\right\} .
$$

Note that

$$
\alpha_{2 i}(\omega)-\alpha_{2 i-1}(\omega) \geq T \quad \text { if } \omega \in\left\{\alpha_{2 i-1}<\infty, \beta_{h}=\infty\right\} \cap \hat{\Omega}_{i} .
$$


Using (3.31) and (3.35), we finally derive that

$$
\begin{aligned}
\infty & >\varepsilon \sum_{i=1}^{\infty} \mathbb{E}\left(I_{\left\{\alpha_{2 i-1}<\infty, \beta_{h}=\infty\right\}}\left[\alpha_{2 i}-\alpha_{2 i-1}\right]\right) \\
& \geq \varepsilon \sum_{i=1}^{\infty} \mathbb{E}\left(I_{\left\{\alpha_{2 i-1}<\infty, \beta_{h}=\infty\right\} \cap \hat{\Omega}_{i}}\left[\alpha_{2 i}-\alpha_{2 i-1}\right]\right) \\
& \geq \varepsilon T \sum_{i=1}^{\infty} \mathbb{P}\left(\left\{\alpha_{2 i-1}<\infty, \beta_{h}=\infty\right\} \cap \hat{\Omega}_{i}\right) \\
& \geq \varepsilon T \sum_{i=1}^{\infty} \varepsilon=\infty,
\end{aligned}
$$

which is a contradiction. Hence, (3.26) must hold. The proof is complete.

\section{Exponential Stabilization}

In the previous section, we have discussed various asymptotic stabilities by feedback controls based on discrete-time state observations. However, all these stabilities do not reveal the rate at which the solution tends to zero. In this section, we will discuss the exponential stabilization by feedback controls. For this purpose, we need to impose another condition.

Assumption 4.1 Assume that there is a pair of positive numbers $c_{1}$ and $c_{2}$ such that

$$
c_{1}|x|^{2} \leq U(x, i, t) \leq c_{2}|x|^{2}
$$

for all $(x, i, t) \in R^{n} \times S \times R_{+}$.

The following theorem shows that the controlled system (2.1) can be stabilized in the sense of both mean square and almost sure exponential stability.

Theorem 4.2 Let Assumptions 2.1, 2.2, 3.1 and 4.1 hold. Let $\tau>0$ be sufficiently small for (3.5) to hold and set

$$
\theta=\frac{K_{3}^{2}}{\lambda_{1}} \quad \text { and } \quad \lambda=\lambda_{2}-\theta \tau\left[2 \tau\left(K_{1}^{2}+2 K_{3}^{2}\right)+K_{2}^{2}\right]
$$

(so $\lambda>0)$. Then the solution of the controlled system (2.1) satisfies

$$
\limsup _{t \rightarrow \infty} \frac{1}{t} \log \left(\mathbb{E}|x(t)|^{2}\right) \leq-\gamma
$$

and

$$
\limsup _{t \rightarrow \infty} \frac{1}{t} \log (|x(t)|) \leq-\frac{\gamma}{2} \quad \text { a.s. }
$$

for all initial data $x_{0} \in R^{n}$ and $r_{0} \in S$, where $\gamma>0$ is the unique root to the following equation

$$
2 \tau \gamma e^{2 \tau \gamma}\left(H_{1}+\tau H_{2}\right)+\gamma c_{2}=\lambda
$$

in which

$$
H_{1}=\theta \tau\left(2 \tau\left(K_{1}^{2}+2 K_{3}^{2}\right)+K_{2}^{2}\right)+\frac{24 \tau^{3} K_{3}^{4}}{1-6 \tau^{2} K_{3}^{2}}, \quad H_{2}=\frac{12 \theta \tau^{2} K_{3}^{2}\left(\tau K_{1}^{2}+K_{2}^{2}\right)}{1-6 \tau^{2} K_{3}^{2}} .
$$


Proof. By the generalized Itô formula, we have

$$
\mathbb{E}\left[e^{\gamma t} V\left(\hat{x}_{t}, \hat{r}_{t}, t\right)\right]=V\left(\hat{x}_{0}, \hat{r}_{0}, t\right)+\mathbb{E} \int_{0}^{t} e^{\gamma z}\left[\gamma V\left(\hat{x}_{z}, \hat{r}_{z}, z\right)+L V\left(\hat{x}_{z}, \hat{r}_{z}, z\right)\right] d z
$$

for $t \geq 0$. Using (3.17), (3.19) and (4.1), we get

$$
c_{1} e^{\gamma t} \mathbb{E}|x(t)|^{2} \leq C_{1}+\int_{0}^{t} e^{\gamma z}\left[\gamma \mathbb{E}\left(V\left(\hat{x}_{z}, \hat{r}_{z}, z\right)\right)-\lambda \mathbb{E}|x(z)|^{2}\right] d z .
$$

Define

$$
\bar{V}\left(\hat{x}_{t}, \hat{r}_{t}, t\right):=\theta \int_{t-\tau}^{t} \int_{s}^{t}\left[\tau\left|f(x(v), r(v), v)+u\left(x\left(\delta_{v}\right), r(v), v\right)\right|^{2}+|g(x(v), r(v), v)|^{2}\right] d v d s .
$$

By (3.1) and (4.1), we then have

$$
\mathbb{E}\left(V\left(\hat{x}_{z}, \hat{r}_{z}, z\right)\right) \leq c_{2} \mathbb{E}|x(z)|^{2}+\mathbb{E}\left(\bar{V}\left(\hat{x}_{z}, \hat{r}_{z}, z\right)\right) .
$$

Moreover, by Assumptions 2.1 and 2.2,

$$
\begin{aligned}
& \mathbb{E}\left(\bar{V}\left(\hat{x}_{z}, \hat{r}_{z}, z\right)\right) \leq \theta \tau \int_{z-\tau}^{z}\left[\left(2 \tau K_{1}^{2}+K_{2}^{2}\right) \mathbb{E}|x(v)|^{2}+2 \tau K_{3}^{2} \mathbb{E}\left|x\left(\delta_{v}\right)\right|^{2}\right] d v \\
& \leq \theta \tau \int_{z-\tau}^{z}\left[\left(2 \tau\left(K_{1}^{2}+2 K_{3}^{2}\right)+K_{2}^{2}\right) \mathbb{E}|x(v)|^{2}+4 \tau K_{3}^{2} \mathbb{E}\left|x(v)-x\left(\delta_{v}\right)\right|^{2}\right] d v .
\end{aligned}
$$

By Theorem 3.2, we see that $\mathbb{E}\left(\bar{V}\left(\hat{x}_{z}, \hat{r}_{z}, z\right)\right)$ is bounded on $z \in[0,2 \tau]$. For $z \geq 2 \tau$, by (3.21), we have

$$
\mathbb{E}\left(\bar{V}\left(\hat{x}_{z}, \hat{r}_{z}, z\right)\right) \leq H_{1} \int_{z-\tau}^{z} \mathbb{E}|x(v)|^{2} d v+H_{2} \int_{z-\tau}^{z} \int_{\delta_{v}}^{v} \mathbb{E}|x(y)|^{2} d y d v .
$$

where both $H_{1}$ and $H_{2}$ have been defined by (4.5). But

$$
\int_{z-\tau}^{z} \int_{\delta_{v}}^{v} \mathbb{E}|x(y)|^{2} d y d v \leq \int_{z-\tau}^{z} \int_{v-\tau}^{v} \mathbb{E}|x(y)|^{2} d y d v \leq \tau \int_{z-2 \tau}^{z} \mathbb{E}|x(y)|^{2} d y .
$$

We hence have

$$
\mathbb{E}\left(\bar{V}\left(\hat{x}_{z}, \hat{r}_{z}, z\right)\right) \leq\left(H_{1}+\tau H_{2}\right) \int_{z-2 \tau}^{z} \mathbb{E}|x(y)|^{2} d y .
$$

Substituting this into (4.8) and then putting the resulting inequality further to (4.6), we get that, for $t \geq 2 \tau$,

$$
\begin{aligned}
c_{1} e^{\gamma t} \mathbb{E}|x(t)|^{2} \leq & C+\gamma\left(H_{1}+\tau H_{2}\right) \int_{2 \tau}^{t} e^{\gamma z}\left(\int_{z-2 \tau}^{z} \mathbb{E}|x(y)|^{2} d y\right) d z \\
& -\left(\lambda-\gamma c_{2}\right) \int_{0}^{t} e^{\gamma z} \mathbb{E}|x(z)|^{2} d z .
\end{aligned}
$$

But

$$
\int_{2 \tau}^{t} e^{\gamma z}\left(\int_{z-2 \tau}^{z} \mathbb{E}|x(y)|^{2} d y\right) d z \leq \int_{0}^{t} \mathbb{E}|x(y)|^{2}\left(\int_{y}^{y+2 \tau} e^{\gamma z} d z\right) d y \leq 2 \tau e^{2 \tau \gamma} \int_{0}^{t} e^{\gamma y} \mathbb{E}|x(y)|^{2} d y .
$$


Substituting this into (4.12) yields

$$
c_{1} e^{\gamma t} \mathbb{E}|x(t)|^{2} \leq C+\left(2 \tau \gamma e^{2 \tau \gamma}\left(H_{1}+\tau H_{2}\right)+\gamma c_{2}-\lambda\right) \int_{0}^{t} e^{\gamma z} \mathbb{E}|x(z)|^{2} d z .
$$

Recalling (4.4), we see

$$
c_{1} e^{\gamma t} \mathbb{E}|x(t)|^{2} \leq C \quad \forall t \geq 2 \tau .
$$

The assertion (4.2) follows immediately. Finally by [23, Theorem 8.8 on page 309], we can obtain the another assertion (4.3) from (4.14). The proof is therefore complete.

\section{Corollaries}

The use of our theorems established in the previous two sections depends on Assumptions 2.1, 2.2, 3.1 and 4.1. Among these, Assumption 3.1 is the critical one as the others can be verified easily. In other words, it is critical if we can design a control function $u(x, i, t)$ which satisfies Assumption 2.2 so that we can then further find a Lyapunov function $U(x, i, t)$ that fulfills Assumption 3.1.

It is known that the stabilization problem (2.1) by the continuous-time (regular) feedback control has been discussed by several authors e.g. [12, 20, 22]. That is, to a certain degree, we know how to design a control function $u(x, i, t)$ which satisfies Assumption 2.2 so that we can then further find a Lyapunov function $U(x, i, t)$ that obeys (3.4). If the derivative vector $U_{x}(x, i, t)$ of this Lyapunov function is bounded by a linear function of $x$, we can then verify Assumption 3.1. This motivates us to propose the following alternative assumption.

Assumption 5.1 Assume that there is a function $U \in C^{2,1}\left(R^{n} \times S \times R_{+} ; R_{+}\right)$and two positive numbers $\lambda_{3}, \lambda_{4}$ such that

$$
\mathcal{L} U(x, i, t) \leq-\lambda_{3}|x|^{2}
$$

and

$$
\left|U_{x}(x, i, t)\right| \leq \lambda_{4}|x|
$$

for all $(x, i, t) \in R^{n} \times S \times R_{+}$.

In this case, if we choose a positive number $\lambda_{1}<\lambda_{3} / \lambda_{4}^{2}$, then

$$
\mathcal{L} U(x, i, t)+\lambda_{1}\left|U_{x}(x, i, t)\right|^{2} \leq-\left(\lambda_{3}-\lambda_{1} \lambda_{4}^{2}\right)|x|^{2} .
$$

But this is the desired condition (3.3) if we set $\lambda_{2}=\lambda_{3}-\lambda_{1} \lambda_{4}^{2}$. In other words, we have shown that Assumption 5.1 implies Assumption 3.1. The following corollary is therefore clear.

Corollary 5.2 All the theorems in Sections 3 and 4 hold if Assumption 3.1 is replaced by Assumption 5.1.

In practice, we often use the quadratic functions as the Lyapunov functions. That is, we use $U(x, i, t)=x^{T} Q_{i} x$, where $Q_{i}$ 's are all symmetric positive-definite $n \times n$ matrices. In this case, Assumption 4.1 holds automatically with $c_{1}=\min _{i \in S} \lambda_{\min }\left(Q_{i}\right)$ and $c_{2}=$ $\max _{i \in S} \lambda_{\max }\left(Q_{i}\right)$. Moreover, condition (5.2) holds as well with $\lambda_{4}=2 \max _{i \in S}\left\|Q_{i}\right\|$. So all we need is to find $Q_{i}$ 's for (5.1) to hold. This motivate us to propose the following another assumption. 
Assumption 5.3 Assume that there are symmetric positive-definite matrices $Q_{i} \in R^{n \times n}$ $(i \in S)$ and a positive number $\lambda_{3}$ such that

$$
\begin{aligned}
2 x^{T} Q_{i}[f(x, i, t) & +u(x, i, t)]+\operatorname{trace}\left[g^{T}(x, i, t) Q_{i}(x, i, t) g(x, i, t)\right] \\
& +\sum_{j=1}^{N} \gamma_{i j} x^{T} Q_{j} x \leq-\lambda_{3}|x|^{2}
\end{aligned}
$$

for all $(x, i, t) \in R^{n} \times S \times R_{+}$.

The following corollary follows immediately from Theorem 4.2.

Corollary 5.4 Let Assumptions 2.1, 2.2 and 5.3. Set

$$
c_{1}=\min _{i \in S} \lambda_{\min }\left(Q_{i}\right), \quad c_{2}=\max _{i \in S} \lambda_{\max }\left(Q_{i}\right), \lambda_{4}=2 \max _{i \in S}\left\|Q_{i}\right\| .
$$

Choose $\lambda_{1}<\lambda_{3} / \lambda_{4}^{2}$ and then set $\lambda_{2}=\lambda_{3}-\lambda_{1} \lambda_{4}^{2}$. Let $\tau>0$ be sufficiently small for (3.5) to hold and set

$$
\theta=\frac{K_{3}^{2}}{\lambda_{1}} \quad \text { and } \quad \lambda=\lambda_{2}-\theta \tau\left[2 \tau\left(K_{1}^{2}+2 K_{3}^{2}\right)+K_{2}^{2}\right]
$$

(so $\lambda>0)$. Then the assertions of Theorem 4.2 hold.

\section{Examples}

Let us now discuss some examples to illustrate our theory.

Example 6.1 We first consider the same example as discussed in Mao [19], namely the linear hybrid SDE

$$
d x(t)=A(r(t)) x(t) d t+B(r(t)) x(t) d w(t)
$$

on $t \geq t_{0}$. Here $w(t)$ is a scalar Brownian motion; $r(t)$ is a Markov chain on the state space $S=\{1,2\}$ with the generator

$$
\Gamma=\left[\begin{array}{rr}
-1 & 1 \\
1 & -1
\end{array}\right]
$$

and the system matrices are

$$
\begin{array}{ll}
A_{1}=\left[\begin{array}{rr}
1 & -1 \\
1 & -5
\end{array}\right], & A_{2}=\left[\begin{array}{rr}
-5 & -1 \\
1 & 1
\end{array}\right], \\
B_{1}=\left[\begin{array}{rr}
1 & 1 \\
1 & -1
\end{array}\right], & B_{2}=\left[\begin{array}{rr}
-1 & -1 \\
-1 & 1
\end{array}\right] .
\end{array}
$$

The computer simulation (Figure 6.1) shows this hybrid SDE is not almost surely exponentially stable.

Let us now design a discrete-time-state feedback control to stabilize the system. Assume that the controlled hybrid SDE has the form

$$
\begin{aligned}
d x(t) & =\left[A(r(t)) x(t)+F(r(t)) G(r(t)) x\left(\delta_{t}\right)\right] d t \\
& +B(r(t)) x(t) d w(t),
\end{aligned}
$$



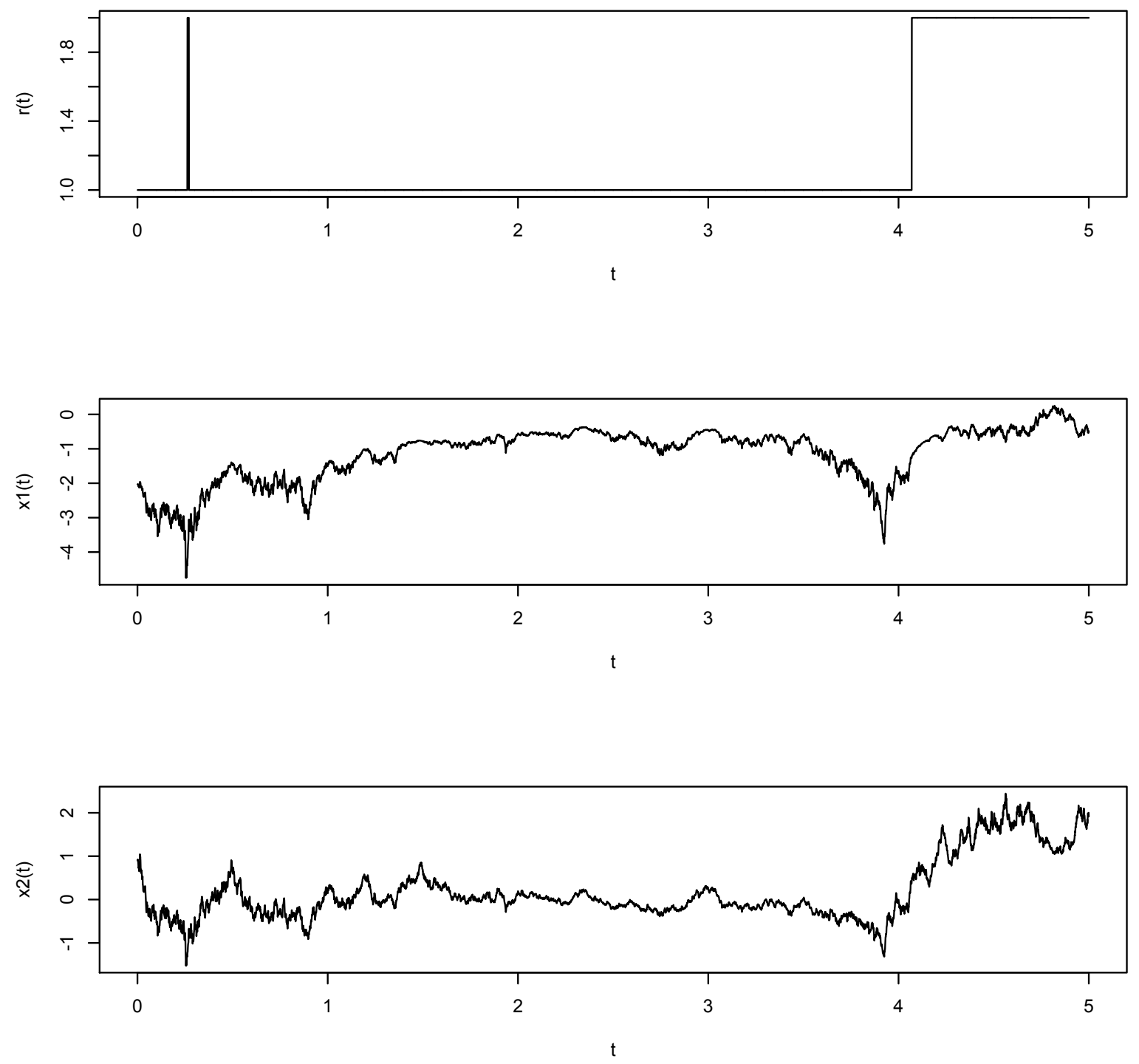

Figure 6.1: Computer simulation of the paths of $r(t), x_{1}(t)$ and $x_{2}(t)$ for the hybrid SDE (6.1) using the Euler-Maruyama method with step size $10^{-6}$ and initial values $r(0)=1, x_{1}(0)=-2$ and $x_{2}(0)=1$.

namely, our controller function has the form $u(x, i, t)=F_{i} G_{i} x$. Here, we assume that

$$
G_{1}=(1,0), \quad G_{2}=(0,1),
$$

and our aim is to seek for $F_{1}$ and $F_{2}$ in $R^{2 \times 1}$ and then make sure $\tau$ is sufficiently small for this controlled SDE to be exponentially stable in mean square and almost surely as well. To apply Corollary 5.4, we observe that Assumptions 2.1 and 2.2 hold with $K_{1}=5.236$ and $K_{2}=\sqrt{2}$. We need to verify Assumption 5.3. It is easy to see the left-hand-side term of (5.4) becomes $x^{T} \bar{Q}_{i} x(i=1,2)$, where

$$
\bar{Q}_{i}:=Q_{i}\left(A_{i}+F_{i} G_{i}\right)+\left(A_{i}^{T}+G_{i}^{T} F_{i}^{T}\right) Q_{i}+B_{i}^{T} Q_{i} B_{i}+\sum_{j=1}^{2} \gamma_{i j} Q_{j} .
$$


Let us now choose $Q_{1}=Q_{2}=I$ (the $2 \times 2$ identity matrix) and

$$
F_{1}=\left[\begin{array}{c}
-10 \\
0
\end{array}\right], \quad F_{2}=\left[\begin{array}{c}
0 \\
-10
\end{array}\right] \text {. }
$$

We then have

$$
\bar{Q}_{1}=\left[\begin{array}{rr}
-16 & 0 \\
0 & -8
\end{array}\right], \quad \bar{Q}_{2}=\left[\begin{array}{rr}
-8 & 0 \\
0 & -16
\end{array}\right] .
$$

Hence, $x^{T} \bar{Q}_{i} x \leq-8|x|^{2}$. In other words, (5.4) holds with $\lambda_{3}=8$. It is also easy to verify that Assumptions 2.1 and 2.2 hold with $K_{1}=5.236, K_{3}=10$ and $K_{2}=\sqrt{2}$. We further compute the parameters specified in Corollary 5.4: $c_{1}=c_{2}=1$ and $\lambda_{4}=2$. Choosing $\lambda_{1}=1$, we then have $\lambda_{2}=4$. Consequently, condition (3.5) becomes

$$
4>200 \tau(227.42 \tau+1), \quad \tau \leq 1 / 40 .
$$

These hold as long as $\tau<0.0074$. By Corollary 5.4, if we set $F_{i}$ as above and make sure that $\tau<0.0074$, then the discrete-time-state feedback controlled hybrid SDE (6.2) is exponentially stable in mean square and almost surely as well. The computer simulation (Figure 6.2) supports this result clearly. It should be pointed out that it is required for $\tau<0.0000308$ in Mao [19], while applying our new theory we only need $\tau<0.0074$. In other words, our new theory has improved the existing result significantly.

Example 6.2 Let us now return to the nonlinear uncontrolled system (2.3). Given that its coefficients satisfy the linear growth condition (2.4), we consider a linear controller function of the form $u(x, i, t)=A_{i} x$, where $A_{i} \in R^{n \times n}$ for all $i \in S$. That is, the controlled hybrid SDE has the form

$$
d x(t)=\left(f(x(t), r(t), t)+A_{r(t)} x\left(\delta_{t}\right)\right) d t+g(x(t), r(t), t) d w(t) .
$$

We observe that Assumption 2.2 holds with $K_{3}=\max _{i \in S}\left\|A_{i}\right\|$. Let us now establish Assumption 5.3 in order to apply Corollary 5.4. We choose $Q_{i}=q_{i} I$, where $q_{i}>0$ and $I$ is the $n \times n$ identity matrix. We estimate the right-hand-side of (5.4):

$$
\begin{aligned}
& 2 x^{T} Q_{i}[f(x, i, t)+u(x, i, t)]+\operatorname{trace}\left[g^{T}(x, i, t) Q_{i}(x, i, t) g(x, i, t)\right]+\sum_{j=1}^{N} \gamma_{i j} x^{T} Q_{j} x \\
& \leq q_{i}\left(2 K_{1}+K_{2}^{2}\right)|x|^{2}+2 q_{i} x^{T} A_{i} x+\sum_{j=1}^{N} \gamma_{i j} q_{j}|x|^{2} \\
& =x^{T}\left(q_{i}\left(2 K_{1}+K_{2}^{2}\right) I+q_{i}\left(A_{i}+A_{i}^{T}\right)+\sum_{j=1}^{N} \gamma_{i j} q_{j} I\right) x .
\end{aligned}
$$

We now assume that the following linear matrix inequalities

$$
q_{i}\left(2 K_{1}+K_{2}^{2}\right) I+Y_{i}+Y_{i}^{T}+\sum_{j=1}^{N} \gamma_{i j} q_{j} I<0
$$

have their solutions of $q_{i}>0$ and $Y_{i} \in R^{n \times n}(i \in S)$. Set $A_{i}=q_{i}^{-1} Y_{i}$ and

$$
-\lambda_{3}=\max _{i \in S} \lambda_{\max }\left(q_{i}\left(2 K_{1}+K_{2}^{2}\right) I+Y_{i}+Y_{i}^{T}+\sum_{j=1}^{N} \gamma_{i j} q_{j} I\right) .
$$



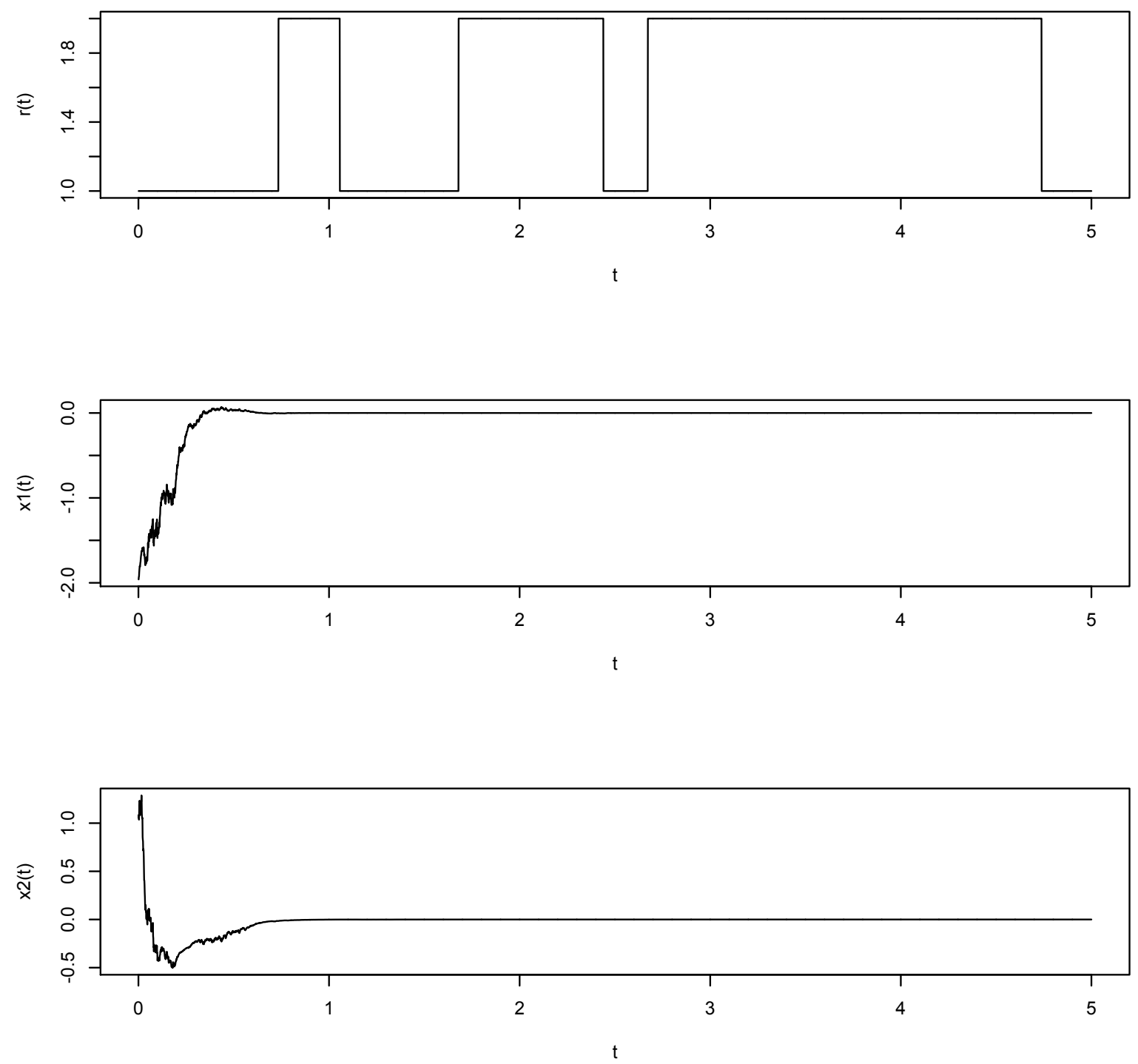

Figure 6.2: Computer simulation of the paths of $r(t), x_{1}(t)$ and $x_{2}(t)$ for the controlled hybrid SDE (6.2) with $\tau=10^{-3}$ using the Euler-Maruyama method with step size $10^{-6}$ and initial values $r(0)=1, x_{1}(0)=-2$ and $x_{2}(0)=1$.

We then see Assumption 5.3 is satisfied. The corresponding parameters in Corollary 5.4 becomes

$$
c_{1}=\min _{i \in S} q_{i}, \quad c_{2}=\max _{i \in S} q_{i}, \quad \lambda_{4}=2 c_{2} .
$$

Choose $\lambda_{1}<\lambda_{3} / \lambda_{4}^{2}$ and then set $\lambda_{2}=\lambda_{3}-\lambda_{1} \lambda_{4}^{2}$. Let $\tau>0$ be sufficiently small for (3.5) to hold. Then, by Corollary 5.4, the controlled system (6.3) is exponentially stable in mean square and almost surely as well. 


\section{Conclusions}

In this paper we have discussed the stabilization of continuous-time hybrid stochastic differential equations by feedback controls based on discrete-time state observations. The stabilities discussed in this paper includes exponential stability and asymptotic stability, in both mean square and almost sure sense, as well as the $H_{\infty}$ stability. One of the significant contributions of this paper is the better bound obtained on the duration $\tau$ between two consecutive state observations. This is achieved by the method of Lyapunov functionals.

\section{Acknowledgements}

The authors would like to thank the editors and referees for their very helpful comments and suggestions. The authors would also like to thank the EPSRC (EP/E009409/1), the Royal Society of London (IE131408), the Royal Society of Edinburgh (RKES115071), the London Mathematical Society (11219), the Edinburgh Mathematical Society (RKES130172), the National Natural Science Foundation of China (11471071), the Natural Science Foundation of Shanghai (14ZR1401200) and the State Administration of Foreign Experts Affairs of China (MS2014DHDX020) for their financial support. Qinwei Qiu would specially like to thank Chinese Scholarship Council to award him the scholarship for him to visit Strathclyde University for one year.

\section{References}

[1] Allwright, J.C., Astolfi, A. and Wong, H.P., A note on asymptotic stabilization of linear systems by periodic, piecewise constant, output feedback, Automatica 41(2) (2005), 339-344.

[2] Anderson, W.J., Continuous-Time Markov Chains, Springer, New York, 1991.

[3] Basak, G.K., Bisi, A. and Ghosh, M.K., Stability of a random diffusion with linear drift, J. Math. Anal. Appl. 202 (1996), 604-622.

[4] Ebihara, Y., Yamaguchi, J. and Hagiwara, T., Periodically time-varying controller synthesis for multiobjective $H_{2} / H_{\infty}$ control of discrete-time systems and analysis of achievable performance, Systems Control Lett. 60(9) (2011), 709-717.

[5] Chammas, A.B. and Leondes, C.T., On the finite time control of linear systems by piecewise constant output feedback, Internat. J. Control 30(2) (1979), 227-234.

[6] Chen,T.W. and Francis, B., Optimal SampledData Control Systems, Springer-Verlag, London, 1995.

[7] Gao, H., Wang, C. and Wang, L., On $H_{\infty}$ performance analysis for continuous-time stochastic systems with polytopic uncertainties, Circuits Systems Signal Processing 24 (2005), 415-429.

[8] Hagiwara,T. and Araki, M., Design of stable state feedback controller based on the multirate sampling of the plant output, IEEE Trans. Automat. Control 33(9) (1988), $812-819$. 
[9] Hagiwara,T. and Araki, M., On preservation of strong stabilizability under sampling. IEEE Trans. Automat. Control 33(11) (1988), 1080-1082.

[10] He, H., Ho, D.W.C. and Lam, J., Stochastic stability analysis of fuzzy Hopfield neural networks with time-varying delays, IEEE Trans. Circuits and Systems II: Express Briefs 52 (2005), 251 -255.

[11] Hu, L., Mao, X. and Shen, Y., Stability and boundedness of nonlinear hybrid stochastic differential delay equations, Systems \&6 Control Letters 62 (2013), 178-187.

[12] Ji, Y. and Chizeck, H.J., Controllability, stabilizability and continuous-time Markovian jump linear quadratic control, IEEE Trans. Automat. Control 35 (1990), $777-$ 788.

[13] Mao, X., Stability of Stochastic Differential Equations with Respect to Semimartingales, Longman Scientific and Technical, 1991.

[14] Mao, X., Exponential Stability of Stochastic Differential Equations, Marcel Dekker, 1994.

[15] Mao X., Stochastic Differential Equations and Their Applications, 2nd Edition, Chichester: Horwood Pub., 2007.

[16] Mao, X., Stability of stochastic differential equations with Markovian switching, Sto. Proc. Their Appl. 79 (1999), 45-67.

[17] Mao, X., Exponential stability of stochastic delay interval systems with Markovian switching, IEEE Trans. Auto. Control 47(10) (2002), 1604-1612.

[18] Mao, X., Stability and stabilization of stochastic differential delay equations, IET Control Theory \& Applications 1(6) (2007), 1551-1566.

[19] Mao,X., Stabilization of continuous-time hybrid stochastic differential equations by discrete-time feedback control, Automatics 49(12) (2013), 3677-3681.

[20] Mao, X., Lam, J. and Huang, L., Stabilisation of hybrid stochastic differential equations by delay feedback control, Systems $\&$ Control Letters 57 (2008), 927-935.

[21] Mao, X., Matasov, A. and Piunovskiy, A.B., Stochastic differential delay equations with Markovian switching, Bernoulli 6(1) (2000), 73-90.

[22] Mao, X., Yin, G. and Yuan, C., Stabilization and destabilization of hybrid systems of stochastic differential equations, Automatica 43 (2007), 264-273.

[23] Mao, X. and Yuan, C., Stochastic Differential Equations with Markovian Switching, Imperial College Press, 2006.

[24] Mariton, M., Jump Linear Systems in Automatic Control, Marcel Dekker, 1990.

[25] Mohammed, S.-E.A., Stochastic Functional Differential Equations, Longman Scientific and Technical, 1986.

[26] Niu, Y., Ho, D.W.C. and Lam, J., Robust integral sliding mode control for uncertain stochastic systems with time-varying delay, Automatica 41 (2005), 873-880. 
[27] Shaikhet, L., Stability of stochastic hereditary systems with Markov switching, Theory of Stochastic Processes 2(18) (1996), 180-184.

[28] Shi, P., Mahmoud, M.S., Yi, J. and Ismail, A., Worst case control of uncertain jumping systems with multi-state and input delay information, Information Sciences 176 (2006), 186-200.

[29] Sun, M., Lam, J., Xu, S. and Zou, Y., Robust exponential stabilization for Markovian jump systems with mode-dependent input delay, Automatica 43 (2007), 1799-1807.

[30] Wei, G., Wang, Z., Shu, H. and Fang, J., Robust $H_{\infty}$ control of stochastic time-delay jumping systems with nonlinear disturbances, Optim. Control Appl. Meth. 27 (2006), $255-271$.

[31] Willsky, A.S. and Levy,B.C., Stochastic stability research for complex power systems, DOE Contract, LIDS, MIT, Report ET-76-C-01-2295, 1979.

[32] Wu, L., Shi, P. and Gao, H., State estimation and sliding mode control of Markovian jump singular systems, IEEE Trans on Automatic Control 55(5) (2010), 1213-1219.

[33] Wu, L., Su, X. and Shi, P., Sliding mode control with bounded $L_{2}$ gain performance of Markovian jump singular time-delay systems, Automatica 48(8) (2012), 1929-1933.

[34] Xu, S., Lam, J., Wang J.L. and Yang, G.H., Stabilization and $H_{\infty}$ control for uncertain stochastic time-delay systems via non-fragile controllers, Asian Journal of Control 8 (2006), 197-200.

[35] Yue, D. and Han, Q., Delay-dependent exponential stability of stochastic systems with time-varying delay, nonlinearity, and Markovian switching, IEEE Trans. Automat. Control 50 (2005), 217-222. 